SOI: $\underline{1.1 / \mathrm{TAS}}$ DOI: $10.15863 / \mathrm{TAS}$

\section{International Scientific Journal Theoretical \& Applied Science}

p-ISSN: 2308-4944 (print) e-ISSN: 2409-0085 (online)

Year: $2017 \quad$ Issue: $09 \quad$ Volume: 53

Published: $30.09 .2017 \quad$ http://T-Science.org

SECTION 2. Applied mathematics. Mathematical modeling.
Sagat Zhunisbekov

doctor of technical Sciences, Professor, academician of the National Engineering Academy of the Republic of Kazakhstan, Professor of the Department «Mechanics and Engineering», ,

Taraz State University named after M.Kh.Dulati

Alexandr Shevtsov candidate of technical sciences, member of PILA (USA), member of European Academy of Natural History (UK), member of Federation of Robotics Kazakhstan, Department of «Mathematics», Deputy Director on Science of faculty of information technologies, automation and telecommunications,

Taraz state University named after M.Kh. Dulati

Danagul Kairliyeva reseacher,

Taraz state University named after M.Kh. Dulati

\title{
ON SOME ASPECTS OF THE IMPLEMENTATION OF THE PRINCIPLE OF HAMILTON IN MAPLE
}

Abstract: The article discusses the opportunities of computer algebra system as applied to the problems with mechanical systems and variational techniques for their solution.

Key words: Maple, variational methods, Hamilton's principle, problems of mechanics.

Language: English

Citation: Zhunisbekov S, Shevtsov A, Kairliyeva D (2017) ON SOME ASPECTS OF THE IMPLEMENTATION OF THE PRINCIPLE OF HAMILTON IN MAPLE. ISJ Theoretical \& Applied Science, 09 (53): 89-92.

Soi: http://s-o-i.org/1.1/TAS-09-53-15 Doi: crossef https://dx.doi.org/10.15863/TAS.2017.09.53.15

\section{Introduction}

Consider a mechanical system considering the interaction between elements of such system are determined by the laws of mechanics[1]. We introduce generalized coordinates that fully define the state of a mechanical system in space[2]. The desired value can take an arbitrary mechanical characteristics[3]. Given the ratio of its differential to the differential at the time as a generalized velocity will get: a set of variables completely determining the state of a mechanical system at all points in time [4-7].

\section{Materials and Methods}

In simple cases the Lagrangian is written as:

$$
L\left(Q, \frac{d Q}{d t}\right)=E_{k}-E_{p},
$$

$E_{k}, E_{p}$ - kinetic and potential energy of the system [1].

Determining on a segment $\left[t_{1}, t_{2}\right]$ the integral of $L$ will receive a functional of the generalized coordinates $Q(t)$.

$$
S[Q]=\int_{t_{1}}^{t_{2}} L\left(Q, \frac{d Q}{d t}\right) .
$$

According to the principle of Hamilton for mechanical systems - if the system is moving according to the laws of mechanics, then $Q(t)$ - is a stationary function for $S[Q]$, or

$$
\frac{d}{d \varepsilon} S[Q+\varepsilon \varphi]_{\varepsilon=0}=0 \text {. }
$$

Hamilton's principle says that of all a priori conceivable trajectories of the system between the times $t_{1}$ and $t_{2}$ is selected the motion of delivering a minimum of an action functional. For tasks with linear movement of the ball [1] have:

$$
\begin{gathered}
L=\frac{m\left(\frac{d r}{d t}\right)^{2}}{2}-k \frac{r^{2}}{2}, \\
S[r]=\int_{t_{1}}^{t_{2}} L\left(r, \frac{d r}{d t}\right) d t=\int_{t_{1}}^{t_{2}}\left[\frac{m}{2}\left(\frac{d r}{d t}\right)^{2}-\frac{k}{2} r^{2}\right] d t .
\end{gathered}
$$




\begin{tabular}{l|lr|ll|ll} 
& ISRA (India) & $=\mathbf{1 . 3 4 4}$ & SIS (USA) & $=\mathbf{0 . 9 1 2}$ & ICV (Poland) & $=\mathbf{6 . 6 3 0}$ \\
Impact Factor: & ISI (Dubai, UAE) $=\mathbf{0 . 8 2 9}$ & PUHЦ (Russia) $=\mathbf{0 . 2 0 7}$ & PIF (India) & $=\mathbf{1 . 9 4 0}$ \\
& GIF (Australia) & $=\mathbf{0 . 5 6 4}$ & ESJI (KZ) & $=\mathbf{3 . 8 6 0}$ & IBI (India) & $=\mathbf{4 . 2 6 0}$ \\
& JIF & $\mathbf{1 1 . 5 0 0}$ & SJIF (Morocco) & $\mathbf{2 . 0 3 1}$ & & \\
\hline
\end{tabular}

$$
\begin{gathered}
S[r+\varepsilon \varphi]=\int_{t_{1}}^{t_{2}}\left[\frac{m}{2}\left(\frac{d(r+\varepsilon \varphi)}{d t}\right)^{2}-\frac{k}{2}(r+\varepsilon \varphi)^{2}\right] d t . \\
\frac{d}{d \varepsilon} S[r+\varepsilon \varphi]=\frac{d}{d \varepsilon} \frac{1}{2} \int_{t_{1}}^{t_{2}}\left[m\left\{\left(\frac{d r}{d t}\right)^{2}+2 \varepsilon \frac{d r}{d t} \frac{d \varphi}{d t}+\varepsilon^{2}\left(\frac{d \varphi}{d t}\right)^{2}\right\}-k\left\{r^{2}+2 \varepsilon r \varphi+\varepsilon^{2} \varphi^{2}\right\}\right] d t= \\
=\int_{t_{1}}^{t_{2}}\left[m\left\{\frac{d r}{d t} \frac{d \varphi}{d t}+\varepsilon\left(\frac{d \varphi}{d t}\right)^{2}\right\}-k\left\{r \varphi+\varepsilon \varphi^{2}\right\}\right] d t .
\end{gathered}
$$

restart;

$x:=t \rightarrow a \cdot \sin \left(\frac{(\mathrm{Pi} \cdot t)}{n}\right) ;$

$$
t \rightarrow a \sin \left(\frac{\pi t}{n}\right)
$$

$x(1)$;

$v:=\operatorname{diff}(x(t), t \$ 1)$

$$
a \sin \left(\frac{\pi}{n}\right)
$$

$$
\frac{a \cos \left(\frac{\pi t}{n}\right) \pi}{n}
$$

$\mathrm{L}:=\mathrm{E}[\mathrm{k}]-\mathrm{E}[\mathrm{p}]$;

$E[k]:=\frac{m \cdot v^{2}}{2} ;$

$$
E_{k}-E_{p}
$$

$$
\frac{1}{2} \frac{m d^{2} \cos \left(\frac{\pi t}{n}\right)^{2} \pi^{2}}{n^{2}}
$$

$E[p]:=m \cdot g \cdot x(t) ;$

$$
m g a \sin \left(\frac{\pi t}{n}\right)
$$

$L$;

$$
\frac{1}{2} \frac{m a^{2} \cos \left(\frac{\pi t}{n}\right)^{2} \pi^{2}}{n^{2}}-m g a \sin \left(\frac{\pi t}{n}\right)
$$

$S:=\operatorname{int}(L, t)$;

$$
\frac{1}{2} \frac{m a^{2} \pi\left(\frac{1}{2} \cos \left(\frac{\pi t}{n}\right) \sin \left(\frac{\pi t}{n}\right)+\frac{1}{2} \frac{\pi t}{n}\right)}{n}+\frac{m g a n \cos \left(\frac{\pi t}{n}\right)}{\pi}
$$

$\operatorname{expand}(S)$;

$$
\frac{1}{4} \frac{m \alpha^{2} \pi \cos \left(\frac{\pi t}{n}\right) \sin \left(\frac{\pi t}{n}\right)}{n}+\frac{1}{4} \frac{m \alpha^{2} \pi^{2} t}{n^{2}}+\frac{m g a n \cos \left(\frac{\pi t}{n}\right)}{\pi}
$$




\begin{tabular}{l|lrl|l|ll} 
& ISRA (India) & $=\mathbf{1 . 3 4 4}$ & SIS (USA) & $=\mathbf{0 . 9 1 2}$ & ICV (Poland) & $=\mathbf{6 . 6 3 0}$ \\
Impact Factor: & ISI (Dubai, UAE) $=\mathbf{0 . 8 2 9}$ & PUHU (Russia) $=\mathbf{0 . 2 0 7}$ & PIF (India) & $=\mathbf{1 . 9 4 0}$ \\
& GIF (Australia) & $\mathbf{0 . 5 6 4}$ & ESJI (KZ) & $=\mathbf{3 . 8 6 0}$ & IBI (India) & $\mathbf{4 . 2 6 0}$
\end{tabular}

$f x:=\operatorname{subs}(a=1, n=0.1, x(t))$;

$f x 1:=\operatorname{subs}(a=2, n=0.1, x(t)) ; f x 2:=\operatorname{subs}(a=5, n=0.1, x(t))$;

$\operatorname{plot}(\{f x, f x 1, f x 2\}, t=0.2) ;$

$$
\begin{gathered}
\sin (10 . \pi t) \\
2 \sin (10 . \pi t) \\
5 \sin (10 . \pi t)
\end{gathered}
$$

$f x:=\operatorname{subs}(a=1, n=0.1, x(t))$;

$f x 1:=\operatorname{subs}(a=1, n=0.5, x(t)) ; f x 2:=\operatorname{subs}(a=1, n=1, x(t))$;

$\operatorname{plot}(\{f x, f x 1, f x 2\}, t=0.2) ;$

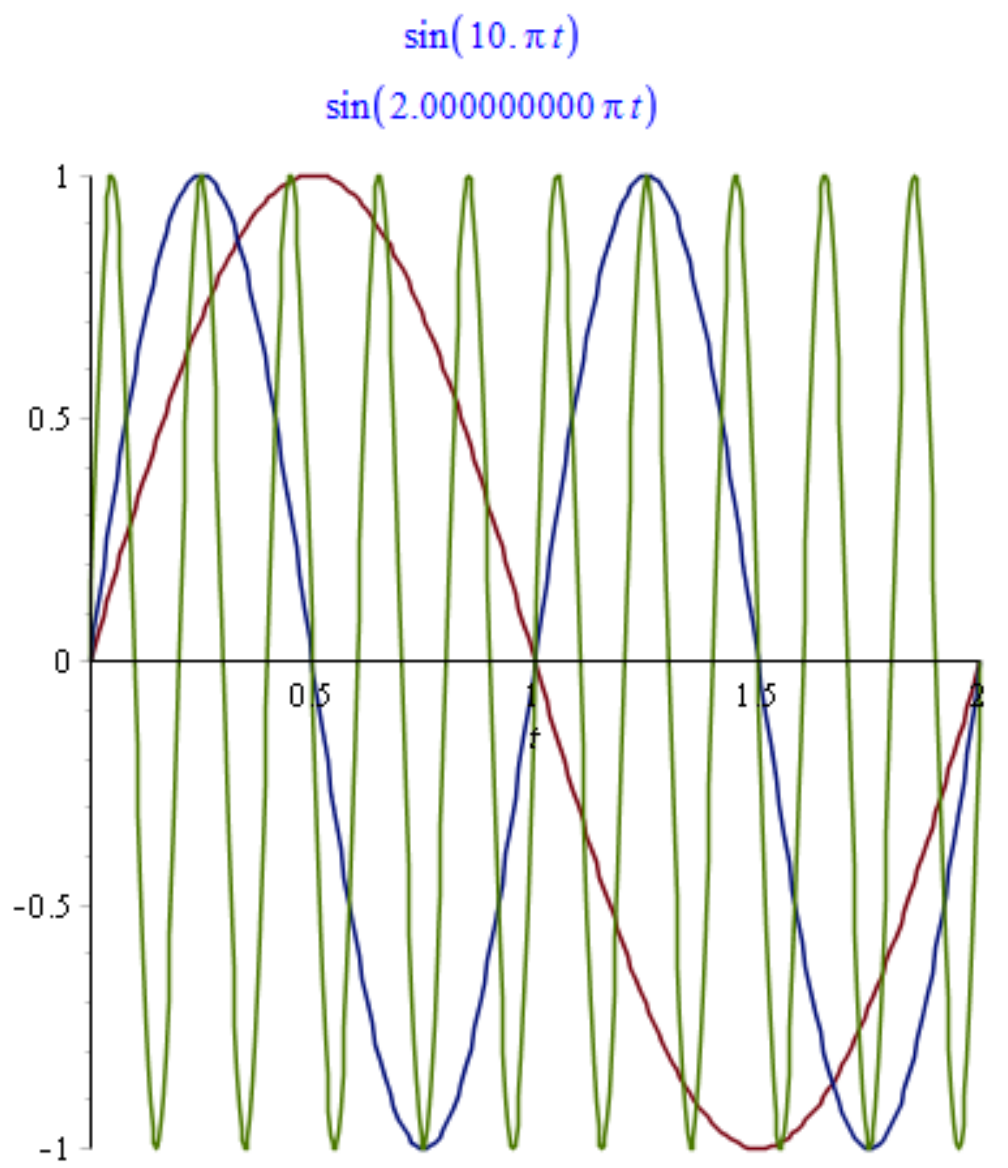

$s x:=(\operatorname{subs}(g=9.8, m=0.01, a=1, n=0.1, S))$;

$s x l:=\operatorname{subs}(g=9.8, m=0.01, a=2, n=0.1, S)$;

$s \times 2:=\operatorname{subs}(g=9.8, m=0.01, a=5, n=0.1, S)$;

\section{Conclusion}

The obtained results demonstrate the behavior of the function actions $S(r)$ based on changing initial conditions in time. 


\begin{tabular}{l|lrl|l|ll} 
& ISRA (India) & $=\mathbf{1 . 3 4 4}$ & SIS (USA) & $=\mathbf{0 . 9 1 2}$ & ICV (Poland) & $=\mathbf{6 . 6 3 0}$ \\
Impact Factor: & ISI (Dubai, UAE) $=\mathbf{0 . 8 2 9}$ & PUHL (Russia) $=\mathbf{0 . 2 0 7}$ & PIF (India) & $=\mathbf{1 . 9 4 0}$ \\
& GIF (Australia) & $\mathbf{0 . 5 6 4}$ & ESJI (KZ) & $=3.860$ & IBI (India) & $=\mathbf{4 . 2 6 0}$ \\
& JIF & $\mathbf{1 . 5 0 0}$ & SJIF (Morocco) & $=\mathbf{2 . 0 3 1}$ & & \\
\hline
\end{tabular}

$\operatorname{plot}(\{s x, s \times 1, s \times 2\}, t=0.2) ;$

$$
\begin{aligned}
& 0.05000000000 \pi\left(\frac{1}{2} \cos (10 . \pi t) \sin (10 . \pi t)+5.000000000 \pi t\right)+\frac{0.0098 \cos (10 . \pi t)}{\pi} \\
& 0.2000000000 \pi\left(\frac{1}{2} \cos (10 . \pi t) \sin (10 . \pi t)+5.000000000 \pi t\right)+\frac{0.0196 \cos (10 . \pi t)}{\pi} \\
& 1.250000000 \pi\left(\frac{1}{2} \cos (10 . \pi t) \sin (10 . \pi t)+5.000000000 \pi t\right)+\frac{0.0490 \cos (10 . \pi t)}{\pi}
\end{aligned}
$$

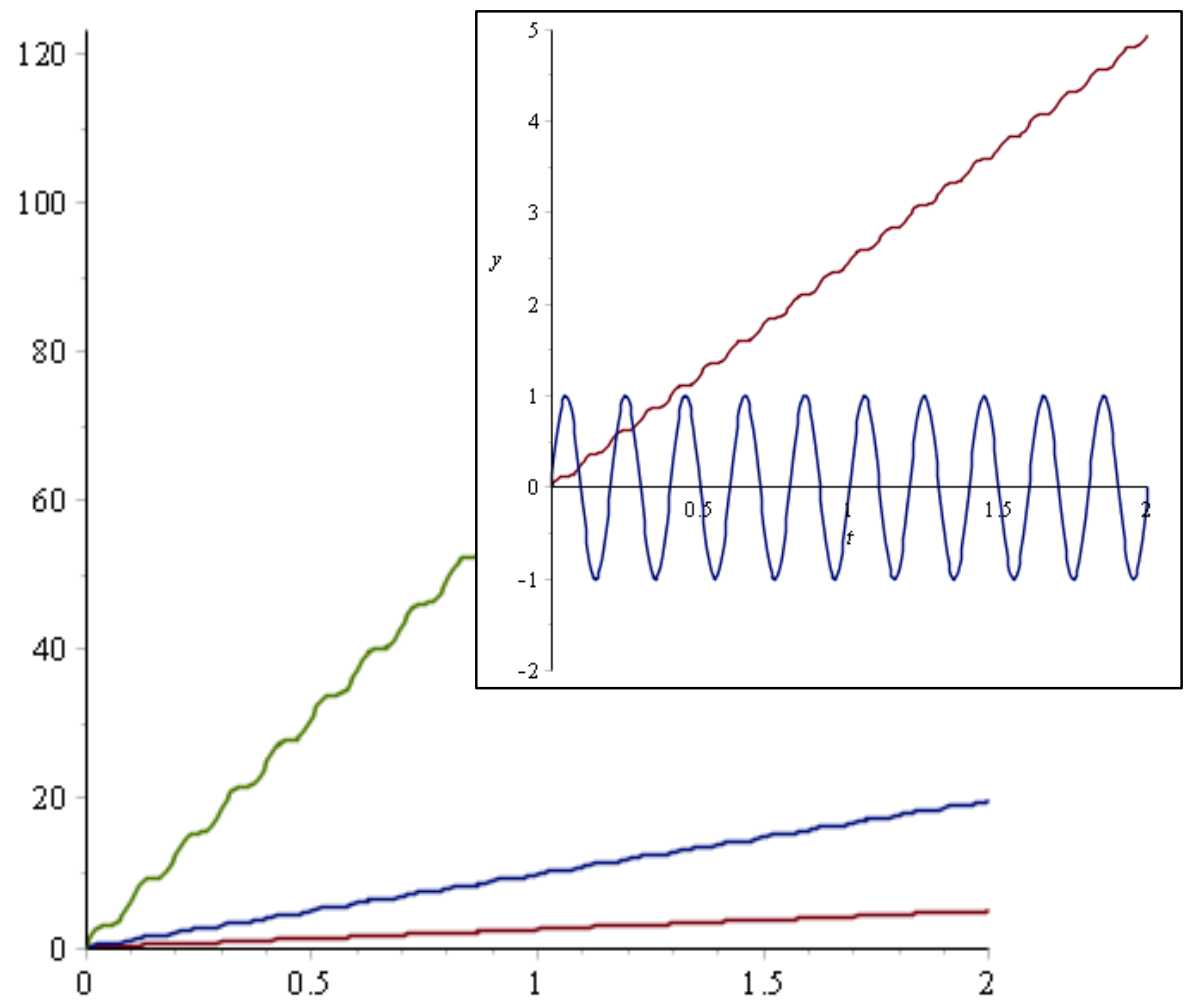

\section{References:}

1. Samarskiy A.A., Mikhaylov A.P. (2001) Matematicheskoe modelirovanie: Idei. Metody. Primery. 2-e izd., ispr. -M.: Fizmatlit. - 320 p.

2. Samarskiy A.A., Mikhaylov A.P. (1987) Komp'yutery i zhizn' (matematicheskoe modelirovanie). -M.: Pedagogika, 1987. -128 p.

3. Samarskiy A.A., Nikolaev E.S. (1978) Metody resheniya setochnykh uravneniy. - M.: Nauka, 1978. - 592 p.

4. Samarskiy A.A., Popov Yu.P. (1980) Raznostnye skhemy gazovoy dinamiki. - M.: Nauka, 1980. - 352 p.

5. Sedov L.I. (11973) Mekhanika sploshnoy sredy. T. I. - M.: Nauka, 1973. - 536 p.

6. Sedov L.I. (1981) Metody podobiya i razmernosti v mekhanike. - M.: Nauka. - 448 p.

7. Sidorov A.F., Shapeev V.P., Yanenko N.N. (1984) Metod differentsial'nykh svyazey i ego prilozheniya $\mathrm{k}$ gazovoy dinamike. Novosibirsk: Nauka, 1984. — 272 p.

8. Silin V.P. (1971) Vvedenie v kineticheskuyu teoriyu gazov. - M.: Nauka, 1971. - 332 p.

9. Tikhonov A.N., Kostomarov D.P. (1984) Vvodnye lektsii po prikladnoy matematike. M.: Nauka, 1984. - 190 p.

10. Tikhonov A.N., Samarskiy A.A. (1972) Uravneniya matematicheskoy fiziki. - M.: Nauka, 1972. - 736 p.

11. Fedorenko R.P. (1994) Vvedenie v vychislitel'nuyu fiziku. M.:Izd-vo MFTI. -526p.

12. Avula X.J.R. (1987) Mathematical Modeling // Encyclopedia of Physical Science. - 1987. V. 7. - P. 719-728.

13. Bender E.A. (1978) An Introduction to Mathematical Modelling. - N.Y.: Wiley, 1978.

14. Cross M. (1985) Learning the Art of Mathematical Modelling. N.Y.: Wiley. - 154 p. 